\title{
Towards an effective model for $(d, p)$ and $(d, n)$ nucleon-transfer reactions to bound states for FLUKA
}

\author{
Francesc Salvat-Pujol ${ }^{1, *}$ and Alfredo Ferrari ${ }^{1}$ on behalf of the FLUKA Collaboration \\ ${ }^{1}$ European Organization for Nuclear Research (CERN) \\ 1 Esplanade des Particules, 1217 Meyrin, Switzerland
}

\begin{abstract}
A general overview is presented of an effective model for the inclusion of $(\mathrm{d}, \mathrm{p})$ and $(\mathrm{d}, \mathrm{n})$ nucleon transfer direct reactions to bound states of the residual nucleus in the general-purpose radiation-transport code FLUKA. The model relies on the distorted-wave Born approximation, employing state-of-the-art subroutines for the numerical solution of the radial Schrödinger equation for the deuteron- and nucleon-nucleus wavefunctions, as well as contemporary optical potential models for the latter two. A final variation of a handful of deuteron optical potential parameters provides the model with additional flexibility and enhances the agreement with experimental nucleon angular distributions in a considerable range of target nuclei and deuteron energies.
\end{abstract}

\section{Introduction}

Deuteron interactions with nuclei at energies below $\sim 200$ $\mathrm{MeV}$ remain a topic of ongoing research [1-5], with eminent practical interest, among other as sources of mostly forward-scattered neutrons and protons, leading to a variety of applications in detector technology, accelerator shielding design, etc. Unfortunately, the inclusion of deuteron interactions in a general-purpose MonteCarlo code for the simulation of radiation transport is not straightforward due to the idiosyncrasies of the deuteron: its low binding energy and the predominantly direct nature of its interactions with target nuclei in the considered energy domain make it not directly amenable to treatment with customary statistical (intranuclear cascade) models.

In this ongoing work, nucleon-transfer reactions of low-energy deuterons $(E \lesssim 200 \mathrm{MeV})$ on a selection of target nuclei of particular relevance for neutronics are addressed. Nucleon transfer from the deuteron to a target nucleus leads to the formation of a recoiling residual nucleus in either the ground state or a low-lying excited state; a nucleon of the conjugate species is emitted. This interaction mechanism leads to a series of discrete sharp peaks in the energy spectrum of the emitted nucleon [6]. The positions of these peaks closely follow the excitation-energy spectrum of the residual nucleus. The angular distribution of the emitted nucleon exhibits a characteristic oscillatory behavior well suited for an effective description based on the distorted-wave Born approximation (DWBA) [7].

In this contribution we present the first stage of our ongoing work towards a DWBA model for the description of $(\mathrm{d}, \mathrm{n})$ and $(\mathrm{d}, \mathrm{p})$ nucleon-transfer reactions to bound states of the residual nucleus. We provide a general overview of the adopted scheme and show that the developed approach

*e-mail: francesc.salvat.pujol@cern.ch is flexible enough to reproduce the available experimental angular distributions of the emitted nucleon. In a future work, a more comprehensive and detailed account will be presented.

\section{The model}

The state-of-the-art package RADIAL for the numerical solution of the radial Schrödinger equation [8] allows one to readily construct distorted waves for the incoming deuteron (using the Han or An optical-potential models $[9,10])$ and for the emitted nucleon (using the Koning and Delaroche potential [11]) in the field of the target and the residual nucleus, respectively. The potential in which the transferred nucleon is bound is modelled as a real WoodsSaxon term plus a spin-orbit coupling term; the depth of the Woods-Saxon term is varied until a binding energy is obtained in agreement with the excitation energy of the nucleus in the final state. The radial integrals arising in the calculation of the transition-matrix element [12] pose no severe numerical issues, inasfar as the bound-nucleon radial function promptly drops to zero outside of the nucleus. Thus, the evaluation of the differential cross section for the emission of a nucleon in a given center-of-mass direction poses no further technical difficulties.

Within the zero-range approximation, and disregarding spin-orbit coupling terms in the optical potential model (OPM) for the time being, the differential cross section for the direct $(\mathrm{d}, \mathrm{n})$ and $(\mathrm{d}, \mathrm{p})$ nucleon-transfer reactions to a bound state $n \ell j$ of the residual nucleus can be expressed 


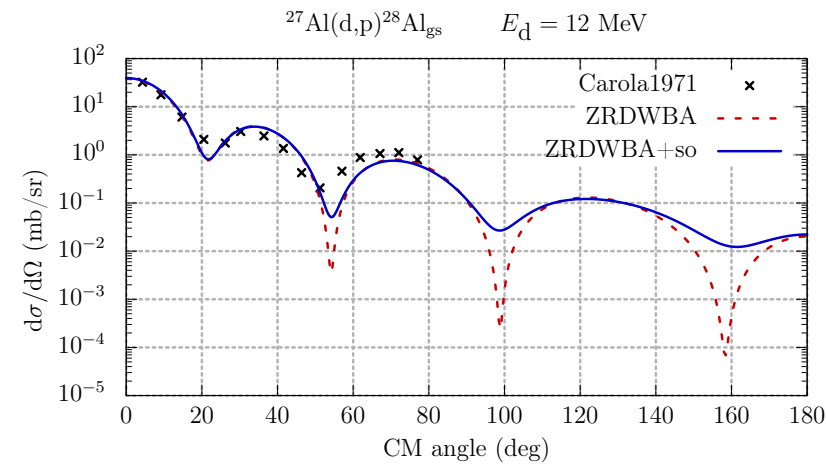

Figure 1. Comparison of the present zero-range DWBA calculation without (red dashed curve) and with (blue solid curve) spin-orbit effects with Carola et al.'s experimental proton angular distribution from the ${ }^{27} \mathrm{Al}(\mathrm{d}, \mathrm{p})_{\mathrm{gs}}$ reaction for $12-\mathrm{MeV}$ deuterons [13].

as [7]:

$$
\begin{aligned}
&\left(\frac{\mathrm{d} \sigma}{\mathrm{d} \Omega^{*}}\right)_{(\mathrm{d}, \mathrm{ej})}=\left(\frac{2 \pi}{\hbar}\right)^{4} \mu_{d} \mu_{e j} \frac{k_{e j}}{k_{d}} \frac{2 I_{A+1}+1}{3\left(2 I_{A}+1\right)} \frac{m_{A+1}}{m_{A}} \\
& \times \sum_{\ell j} \frac{6 \pi^{2} \hbar^{4} D_{0}^{2}}{\mu_{n p}^{2}} S_{n \ell j} \frac{1}{2 \ell+1} \\
& \times \sum_{m} \mid \int \mathrm{d}^{3} \boldsymbol{r} \chi_{\mathrm{ej}}^{(-) *}\left(\boldsymbol{k}_{\mathrm{ejk}}, \frac{m_{A}}{m_{A+1}} \boldsymbol{r}\right)^{2} \\
&\left.Y_{\ell m}^{*}(\hat{\boldsymbol{r}}) \frac{1}{r} P_{n \ell j}(r) \chi_{\mathrm{d}}^{(+)}\left(\boldsymbol{k}_{\mathrm{d}}, \boldsymbol{r}\right)\right|^{2},
\end{aligned}
$$

where the various $\mu$ represent reduced masses for projectile-nucleus combinations, $d$ and " $e j$ " stand for deuteron and ejectile ( $\mathrm{p}$ or $\mathrm{n}$ as needed) respectively, $k$ are CM wavevectors, $I$ are nuclear spins, $A$ and $A+1$ denote the target and the residual nucleus, respectively, $m$ denotes nuclear masses, $D_{0}=124 \mathrm{MeV} \mathrm{fm}^{3 / 2}$ is the zero-range constant, $S_{n \ell j}$ are spectroscopic factors, the $\chi(\boldsymbol{r})$ denote the distorted waves of the incoming deuteron and the outgoing ejectile, $Y_{\ell m}(\hat{\boldsymbol{r}})$ denote spherical harmonics and, finally, $P_{n \ell j}(r)$ are the radial functions of the transferred nucleon.

Figure 1 compares the experimental angular distribution [13] of protons emitted from the reaction ${ }^{27} \mathrm{Al}(\mathrm{d}, \mathrm{p})_{\mathrm{gs}}$ for $12-\mathrm{MeV}$ deuterons (symbols) with the differential cross section (red dashed curve) evaluated as per Eq. (1), disregarding spin-orbit coupling terms in the deuteron and proton OPM. The minima in the calculated cross section are at the correct position, but they are too deep and narrow. Instead, if spin-orbit terms of the potential are properly accounted for [12], the agreement with the experimental data improves substantially (blue solid curve).

The amplitude and diffusiveness of the real WoodsSaxon term of the deuteron OPM, as well as the radius of the imaginary surface term, have been allowed to vary with respect to the values prescribed by Han et al. [10]. This has provided the model with additional flexibility to adapt to the experimental data. Figure 2 displays the con-

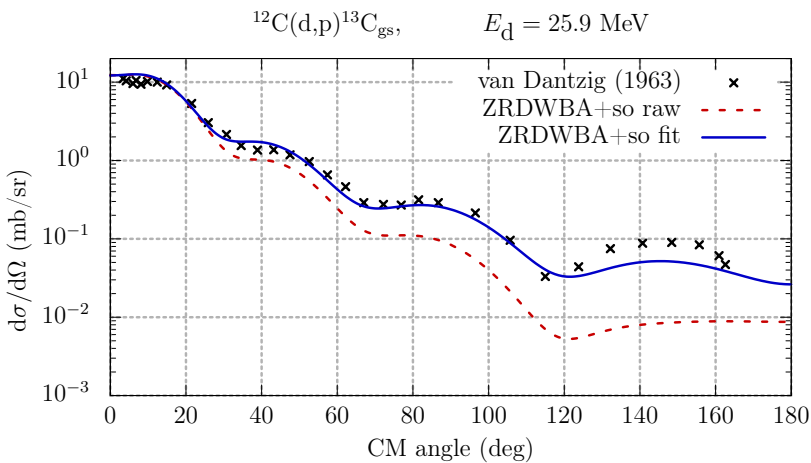

Figure 2. (Symbols) Van Dantzig et al.'s experimental angular distribution of protons emitted from the reaction ${ }^{12} \mathrm{C}(\mathrm{d}, \mathrm{p})_{\mathrm{gs}}$ for 25.9-MeV deuterons [14]. The red dashed and blue solid curves display the calculated differential cross section (including spinorbit effects) with default and with varied deuteron OPM parameter values, respectively.

siderable improvement of the agreement with experimental data when these parameters are allowed to vary within $20 \%$ of their original value, for the angular distribution of protons emitted from the ${ }^{12} \mathrm{C}(\mathrm{d}, \mathrm{p})_{\mathrm{gs}}$ reaction for $25.9-\mathrm{MeV}$ deuterons [14].

The scheme outlined in this work is currently being applied to a broad selection of target isotopes, both for the $(d, p)$ and the $(d, n)$ reactions and for several final states. The availability of evaluated experimental data via EXFOR for a broad selection of isotopes in a considerable energy range (from threshold up to several tens of megaelectronvolt) renders the scheme described here feasible and sufficient for typical Monte Carlo simulation needs.

\section{Summary}

A DWBA calculation scheme has been implemented to include $(\mathrm{d}, \mathrm{p})$ and $(\mathrm{d}, \mathrm{n})$ nucleon transfer direct reactions in the general-purpose radiation transport code FLUKA $[15,16]$. The model yields a reasonable description of the shape of experimental nucleon angular distributions. In spite of the conceptual simplicity of the calculation scheme, the resulting differential cross sections are sufficiently realistic for Monte Carlo simulation needs. The main practical difficulty in the adopted approach lies in the fact that the naturally arising spectroscopic factors need to be adjusted to experimental data (and that a variation of a handful of deuteron OPM parameters is necessary for a final enhancement of the agreement with experimental nucleon angular distributions). Thus, considerable effort is being undertaken to take into account experimental angular distributions to gauge the normalization of our DWBA differential cross sections in as broad a deuteron-energy range as the published data allow. Further details of the model and a more comprehensive overview of the examined cases will be presented in an upcoming dedicated work. 


\section{References}

[1] M. Zadro, Phys. Rev. C 66, 034603 (2002)

[2] T. Ye, S. Hashimoto, Y. Watanabe, K. Ogata, M. Yahiro, J. Kor. Phys. Soc. 59, 871 (2011)

[3] Y. Watanabe, T. Ye, K. Ogata, EPJ Web Conf. 2, 11003 (2010)

[4] S. Nakayama, S. Araki, Y. Watanabe, O. Iwamoto, T. Ye, K. Ogata, Nucl. Dat. Sheets 118, 305 (2014)

[5] B.V. Carlson, R. Capote, M. Sin, EPJ Web Conf. 146, 12001 (2017)

[6] J. Lang, J. Liecthi, R. Muller, P.A. Schmelzbach, J. Smyrski, M. Godlewski, L. Jarczyk, A. Stralkowski, H. Witala, Nucl. Phys. A 477, 77 (1988)

[7] P. Fröbrich, R. Lipperheide, Theory of Nuclear Reactions (Clarendon Press, 1996), ISBN 0198537832
[8] F. Salvat, J.M. Fernández-Varea, Comput. Phys. Commun. 240, 165 (2019)

[9] H. An, C. Cai, Phys. Rev. C 73, 054605 (2006)

[10] Y. Han, Y. Shi, Q. Shen, Phys. Rev. C 74, 044615 (2006)

[11] A.J. Koning, J.P. Delaroche, Nucl. Phys. A 713, 231 (2003)

[12] L. Titus, Ph.D. thesis, Michigan State University (2018)

[13] T.P.G. Carola, J.G. van der Baan, Nucl. Phys. A 173, 414 (1971)

[14] R. van Dantzig, L.A.C. Koerts, Nucl. Phys. 48, 177 (1963)

[15] A. Ferrari, P.R. Sala, A. Fassò, J. Ranft, CERN2005-10, INFN/TC_05/11, SLAC-R-773 (2005)

[16] G. Battistoni, T. Boehlen, F. Cerutti, P.W. Chin, L.S. Esposito, A. Fassò, A. Ferrari, A. Lechner, A. Empl, A. Mairani et al., Ann. Nucl. Energy 82, 10 (2015) 